\title{
On the fundamental relation of laser schlieren deflectometry for temperature measurements in filamentary plasmas ${ }^{\star}$
}

\author{
Jan Schäfer ${ }^{1, a}$, Zdeněk Bonaventura ${ }^{2}$, and Rüdiger Foest ${ }^{1}$ \\ ${ }^{1}$ Leibniz Institute for Plasma Science and Technology, Felix-Hausdorff-Str. 2, 17489 Greifswald, Germany \\ ${ }^{2}$ Department of Physical Electronics, Faculty of Science, Masaryk University, Kotlářská 2, 61137 Brno, Czech Republic
}

Received: 12 December 2014 / Received in final form: 18 February 2015 / Accepted: 26 February 2015 Published online: 15 July 2015 - (C) The Author(s) 2015

\begin{abstract}
Recently, laser schlieren deflectometry (LSD) had been successfully employed as a temperature measurement method to reveal the heat convection generated by micro filaments of a self-organized nonthermal atmospheric plasma jet. Based on the theory of the temperature measurements using LSD, in this work, three approaches for an application of the method are introduced: (i) a hyperbolic-like model of refractive index is applied which allows an analytical theory for the evaluation of the deflection angle to be developed, (ii) a Gaussian shape model for the filament temperature is implemented which is analyzed numerically and (iii) an experimental calibration of the laser deflection with a gas mixture of helium and argon is performed. Thus, these approaches demonstrate that a universal relation between the relative maximum temperature of the filament core $\left(T_{1} / T_{0}\right)$ and a the maximum deflection angle $\delta_{1}$ of the laser beam can be written as $T_{1} / T_{0}=\left(1-\delta_{1} / \delta_{0}\right)^{-1}$, where $\delta_{0}$ is a parameter that is defined by the configuration of the experiment and by the assumed model for the shape of the temperature profile.
\end{abstract}

\section{Introduction}

Atmospheric pressure microdischarges are widely used for diverse material processing [1]. The temperature profile of a contracted filament affects the energetic balance. Hence, its knowledge can help to enhance the efficiency of technological applications. Moreover, many plasma kinetic reactions such as ion conversion or dissociative recombination depend significantly on the neutral gas temperature [2]. Finally, the neutral gas temperature determines the local particle density and therefore any reaction rate. Nevertheless, the local development of the neutral gas temperature in filamentary plasmas at atmospheric pressure often evades from exact determination by most experimental methods. The limitations of the methods are inherently caused by the nature of the filaments which exhibit submillimeter dimensions and steep gradients of the temperature field. The information about the temperature can be obtained using advanced modeling as shown e.g., in [3], where a streamer channel of a dielectric barrier discharge is characterized. The study demonstrates the need of an experimental method, which can explore the

\footnotetext{
${ }^{a}$ e-mail: jschaefer@inp-greifswald.de

* Contribution to the topical issue "The 14th International Symposium on High Pressure Low Temperature Plasma Chemistry (HAKONE XIV)", edited by Nicolas Gherardi, Ronny Brandenburg and Lars Stollenwark

Supplementary online material is available in electronic form at www.epjap.org
}

temperature fields in submilimeter dimensions and in submicrosecond scale. However, up to now, no experimental method satisfies these requirements fully [4]. Invasive experimental methods, where the probe is immersed in the plasma, consider an interaction between the plasma and the surface of the probe itself. Examples are fluorooptic or calorimetric probes (e.g., [5]) that evaluate the local equilibrium between the probe and the surrounding plasma. Disregarding the contacting or monitoring of the probe as an additional difficulty, such methods are slowed down due to time delay needed to attain thermodynamic equilibrium and also the spacial resolution remains poor due to the probe dimension.

As non-contact method, e.g., planar laser-induced fluorescence [6] can be applied. Yet, this method is limited to the emitting plasma areas and moreover the data acquisition often requires a substantial time. Other thermo-visualization methods like infrared thermography [7], Rayleigh scattering [8], schlieren imaging [9,10] or rainbow schlieren deflectometry $[11,12]$ provide a decent spatial characterization, but again a longer signal accumulation is necessary for the measurement of a weakly ionized and minuscule filamentary plasmas. Up to now, the usage of these conventional methods is designed preferably for systems with a substantial amount of energy or a sufficiently high gradient of refractive index, e.g., shock wave unsteadiness or high-power plasmas $[13,14]$.

Laser schlieren deflectometry (LSD) represents a noninvasive diagnostic technique, by which the challenging 
measurement and characterization of neutral gas temperature in the microscopic time and space scales in RF plasma jets can be mastered [15]. In that study [15], an analytically feasible approach is described. The procedure is based on the assumption of a hyperbolic-like profile of the refractive index for a single filament and demonstrates a sufficient agreement with experimental data. The concept of the measurements can be used for advanced modelling of the profile which reflects physical effects behind the temperature field (e.g., plasma heating, heat transfer, etc.). Therefore, in this work, we implemented a temperature profile with a Gaussian shape for the single filament in the methodical concept of LSD, and solved the total deflection of the laser beam numerically. The numerical modelling of the ray propagation trough the temperature field of the filament led to the generalized calibration relation for the calculation of the filament core temperature from the maximum deflection angle obtained by LSD. Additionally, the optical refraction has been calibrated with a gas mixture of helium and argon, thus the relation between the core temperature and maximum deflection has been obtained experimentally. Surprisingly, results of all three approaches can be expressed with a single formula, describing LSD universally.

\section{Experimental set-up}

\subsection{Plasma source}

The experimental set-up (Fig. 1) investigated here is a non-thermal plasma jet [1]. It features two ring electrodes attached to the outer quartz capillary $\left(D_{\text {in }}=4.0 \mathrm{~mm}\right)$. The upper electrode is coupled to the RF generator $(27.12 \mathrm{MHz})$ via a matching network. The applied RF power was fixed at $10 \mathrm{~W}$ for the purpose of this study. The discharge is fed with argon (5.0) with a fixed flow rate of $0.8 \mathrm{slm}$. Inside the outer capillary, a second capillary is inserted in order to dose organic vapors for optional thin film deposition. Depending on the operation parameter flow rate vs. power, different regimes of the plasma jet can be established. The self-organized regime (locked mode, LM) of the jet has been chosen as a prototypical regime in this study. The characteristic of the LM is an equidistant location of all filaments around the inner wall of the outer capillary and a steady azimuthal rotation of the filaments around the axis of the capillary. Throughout this study the experiment was operated in the LM of 3 filaments (LM3).

\subsection{Laser schlieren deflectometer}

The LSD set-up consists of a He-Ne laser (Linos) and a high-speed CMOS camera (Photon Focus). The displacement of the laser spot on the image sensor (pixel size: $8 \mu \mathrm{m}, 1312 \times 1082$ pixels) of the camera is monitored directly. The laser beam (classified as $3 \mathrm{~A}, 632.82 \mathrm{~nm}, \mathrm{cw}$ ) is led perpendicular through the effluent region, close to the nozzle of the capillary $(z=0 \mathrm{~mm})$. The output power of the laser $(0.6 \mathrm{~W})$ is sufficiently low to avert any influence

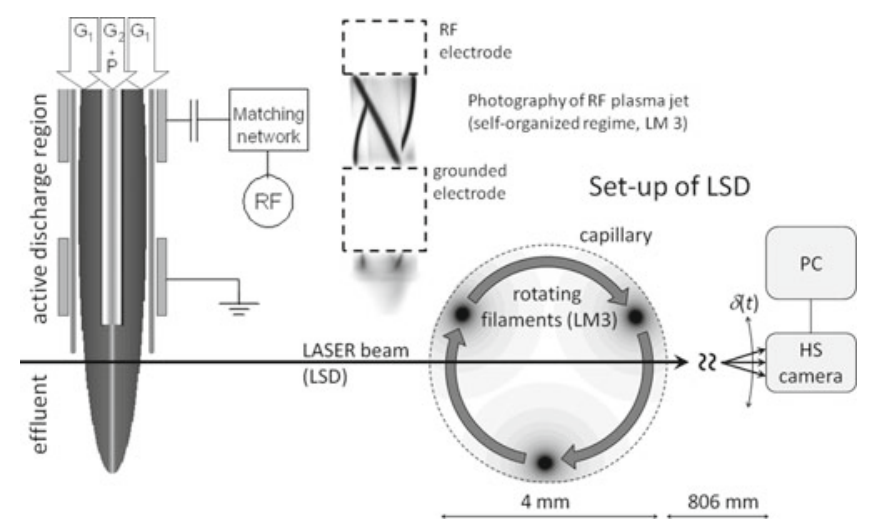

Fig. 1. Experimental set-up of the RF plasma jet and LSD with an inverted photography of the jet (LM3).

of the beam on the properties of the plasma jet. Deflection of the laser beam due to refraction in the gradient field of the refractive index is recorded at a distance of $80.6 \mathrm{~cm}$ from the plasma jet at $10 \mathrm{kHz}$ sample frequency. The laser beam is specified by the spot diameter of $490 \mu \mathrm{m}$ and beam divergence of $1.7 \mathrm{mrad}$. The parameters of the beam enable to characterize filament structures with diameters down to approx. $100 \mu \mathrm{m}$. The principle of the LSD is valid, if the dimension of the central area of the laser spot is smaller or comparable with the characteristic dimension of the refractive structure. Thus, the mean position of the intensity distribution can be traced quickly. In the opposite case, if the laser spot is a magnitude larger than the characteristic dimension, then the interaction of the beam with the refractive structure leads to the usual projection of a schlieren image on the camera chip. In this case, the evaluation would require a more detailed consideration of the imaged intensity distribution.

\section{Analytical approach}

The trajectory tracing of an optical ray in a cylindrically symmetric profile of the refractive index is an elementary task having several analogies in physics. A typical example is the calculation of atmospheric refraction in astronomy. Based on Fermat's principle or on Snell's law, the angular deflection $\delta=\delta(\rho)$ of the ray can be derived for the diminutive scale of a plasma filament, too [16]:

$$
\delta=\pi-2 \int_{r_{0}}^{\infty} \frac{\mathrm{d} r}{r \sqrt{\left(\frac{r}{\rho} f\right)^{2}-1}},
$$

where $r$ is the radial coordinate related to the centre of the refractive index profile $n(r), r_{0}$ is the closest distance to the centre $r=0, \rho$ is the impact distance (for the singular case of spatially constant refractive index: $\left.\rho=r_{0}\right)$ and $f$ is a shape function of the refractive index profile defined by $f=n(r) / n_{0}$, where $n_{0}$ is the surrounding refractive index. Note that the same equation (1) describes the Rutherford scattering of $\alpha$ particles with an initial energy $E_{0}$ approaching the centre of an atom on the potential level $U$, if $f$ in equation (1) is replaced by $\sqrt{\left(E_{0}-U\right) / E_{0}}$ [17]. 
We solved equation (1) for a hyperbole-like profile [15], assuming a constant refractive index $n_{1}$ in the filament core characterized with radius $r_{\mathrm{c}}$ :

$$
\begin{array}{ll}
f(r)=\frac{n_{1}}{n_{0}} & \text { for } r \leq r_{\mathrm{c}} \\
f(r)=1-\frac{r_{\mathrm{c}}\left(n_{0}-n_{1}\right)}{n_{0} r} & \text { for } r>r_{\mathrm{c}} .
\end{array}
$$

The integrated function $\delta(\rho)$ has two local extrema characterized with a deflection amplitude $\delta_{1}$ :

$$
\delta_{1}=2 \frac{n_{0}-n_{1}}{n_{1}} .
$$

If the neutral gas temperature at atmospheric pressure is the only factor determining the refractive index of the gas, then the Arago-Biot relation [18] (called Landolt's law, too [19]) can be written for the discussed system:

$$
n_{1}=1+\frac{T_{0}}{T_{1}}\left(n_{0}-1\right),
$$

where $T_{0}$ is the surrounding temperature of the gas and $T_{1}$ is the temperature in the filament core. The combination of equations (3) and (4) leads to the principle equation of LSD, which links the maximum deflection angle $\delta_{1}$ to the maximum temperature $T_{1}$ in the centre of the plasma filament:

$$
\frac{T_{1}}{T_{0}}=\left(1-\frac{\delta_{1}}{2\left(n_{0}-1\right)}\right)^{-1} .
$$

\section{Calibration}

LSD can be calibrated either by an independent measurement of the varying temperature directly or by an isothermal variation of refractive index combined with equation (4) indirectly. For the purpose of exact calibration, the second method has been chosen, because no alternative temperature measurements with comparable accuracy to the LSD are standardized for the investigated system up to now. Therefore, a directed cylindrical gas flow of a helium and argon mixture was prepared. The gas mixture is introduced into a hollow needle with an inner diameter of $800 \mu \mathrm{m}$. Thus, the cylindrically symmetric gas stream is characterized by the given refractive index in the centre of the stream determined by the ratio of the helium and argon concentration. An advantage of the mixture is that it can emulate the transition of optical properties from ambient air condition (100\% argon) towards vacuum (100\% helium). Thus the temperature interval from laboratory temperature up to $2400 \mathrm{~K}$ can be calibrated with an exceptional accuracy. The results of the experiment demonstrate a linear dependence of the maximum deflection angle on the mixing ratio and therefore on the refractive index, too (see Fig. 2):

$$
\delta_{1}=A-B \frac{n-n(\mathrm{He})}{n(\mathrm{Ar})-n(\mathrm{He})},
$$

where $n(\mathrm{He})=1.000032$ and $n(\mathrm{Ar})=1.00026$ are refractive indexes at the laboratory temperature of $298 \mathrm{~K}$,

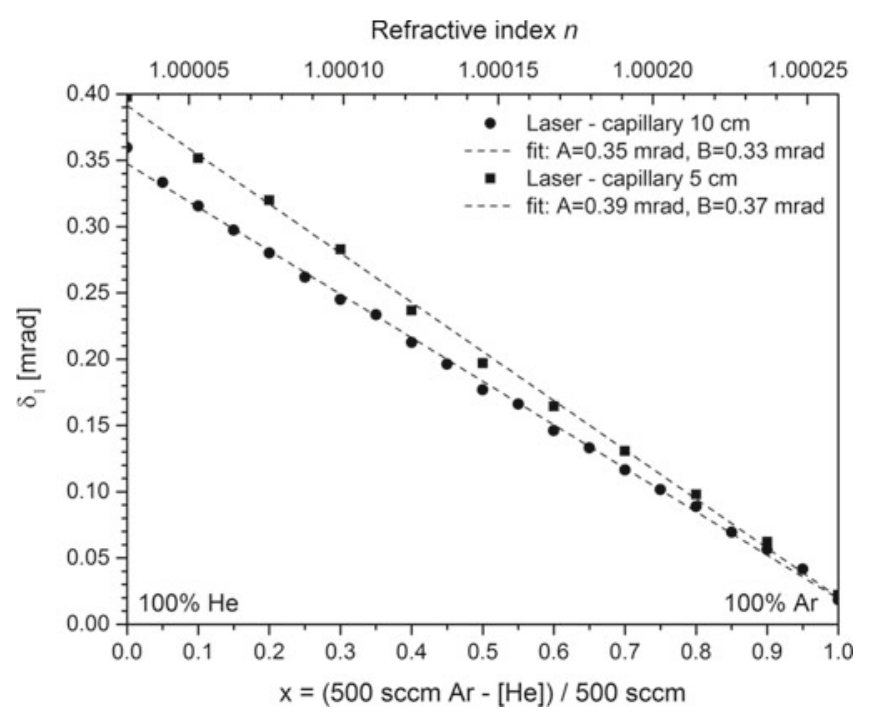

Fig. 2. Maximum deflection of the laser beam by neutral gas stream of a helium-argon mixture. Measurements for two configurations (distance of the capillary from the laser), diameter of the capillary $800 \mu \mathrm{m}$, ambient gas - air, $298 \mathrm{~K}, 20 \%$ humidity, data fitted by $\delta_{1}=A-B x, R^{2}=0.998$.

$n$ is the refractive index of the mixture (note that $n($ air $)=1.00027$ ), and $A$ and $B$ are the fitting parameters obtained by linear regression of the measured data points. The observed linearity is characterized by a surprisingly high predictive power of the linear fit $R^{2}=0.998$ in this measurement. Moreover, the ratio $A / B$ equals 1 with an accuracy better than $5 \%$. Combining equations (6) and (4), the calibration relation for the temperature can be derived (assumed $n_{0}$ in equation (4) is $n(\mathrm{Ar})$ ):

$$
\frac{T_{1}}{T_{0}}=\frac{1}{\frac{n(\mathrm{He})+\frac{A}{B}(n(\mathrm{Ar})-n(\mathrm{He}))-1}{n(\mathrm{Ar})-1}-\frac{n(\mathrm{Ar})-n(\mathrm{He})}{(n(\mathrm{Ar})-1) B} \delta_{1}} .
$$

Considering the approximations $n(\mathrm{He}) \approx 1$ and $A / B \approx 1$ equation (7) can be rewritten:

$$
\frac{T_{1}}{T_{0}}=\left(1-\frac{\delta_{1}}{B}\right)^{-1}
$$

where only the fitting parameter $B$ determines the calibration equation. The constant $B$ depends on the configuration of the calibration experiment, particularly on the diameter of the gas stream and on the cross-section of the laser spot at the position of the gas stream. If the experimentally obtained calibration equation (8) shall be used for the temperature determination, then these proportions should be chosen as close as possible to the parameter of the real experiment with the plasma jet.

\section{Numerical approach}

In this section we describe the numerical approach used in order to determine the maximum deflection angle for the models for which analytical attempts would rapidly 
become intractable or even impossible. In a sense, our approach resembles forward ray tracing. In principle, the classical ray tracing algorithms assumes - for numerical efficiency - that the medium is segmented into subdomains with constant index of refraction in which the light rays propagate along straight lines and Fresnel equations are used on the interfaces between individual sub-domains to ensure consistency for refraction angles. For example POV-ray [20] allows to account for multiple reflections in arbitrarily shaped refractive objects for which correctness for scientific problems has been already successfully verified [21]. However, media with gradient refractive index represent another class of problems for which classical ray tracing tools become very inefficient. Indeed, it would require a prohibitively large number of sub-domains to mimic spatial gradients of refractive index with sufficient precision.

Therefore our approach sticks to tracing individual rays from a source plain through the media towards the target plain solving numerically the differential equation for the light ray:

$$
\frac{\mathrm{d}}{\mathrm{d} s}\left(n \frac{\mathrm{d} \mathbf{r}}{\mathrm{d} s}\right)=\nabla n
$$

where $\mathbf{r}$ is a position vector of a point on a ray, $s$ is an arc length of a ray and $n=n(\mathbf{r})$ is the refractive index [24].

If we choose the stepping parameter $a$ such that:

$$
|\mathrm{d} \mathbf{r} / \mathrm{d} a|=n
$$

then equation (9) becomes

$$
\frac{\mathrm{d}^{2} \mathbf{r}}{\mathrm{d} a^{2}}=n \nabla n=\nabla\left(\frac{1}{2} n^{2}\right) .
$$

Note that this equation is a form of Newton's second law (for a dimensionless unit mass) with $\frac{1}{2} n^{2}$ being an analogue of the potential energy [25]. Equation (10) can then be solved as initial value problem:

$$
\begin{aligned}
& \frac{\mathrm{d} \mathbf{v}}{\mathrm{d} a}=n(\mathbf{r}) \nabla n(\mathbf{r}), \\
& \frac{\mathrm{d} \mathbf{r}}{\mathrm{d} a}=\mathbf{v},
\end{aligned}
$$

with initial condition

$$
\mathbf{r}(0)=\mathbf{r}_{0} \quad \text { and } \quad|\mathbf{v}(0)|=n\left(\mathbf{r}_{0}\right) .
$$

Numerical solution of (11)-(13) is performed by lsode solver from ODEPACK [22] interfaced by GNU Octave [23].

Sample rays are initiated at $\mathbf{r}=\left(\xi_{i},-1.0\right) \mathrm{m}$ with sufficiently fine distribution of $\xi_{i}$ along the source plane ensuring thus that the correct value of the maximal deflection angle is captured. The deflection angle is calculated as $\delta=\operatorname{atan} 2\left(v_{x}, v_{y}\right)$, where $v_{x}$ and $v_{y}$ are components of $\mathbf{v}$ on the target plain $y=1.0 \mathrm{~m}$. Relative and absolute tolerances of the solver are set to a tiny value of $10^{-12}$ to ensure the desirable solution accuracy of a possibly stiff system. Moreover the maximum step size is set to $10^{-4}$ assuring that the small filament core - compared to the source plane to target plane distance - is not skipped on the course of adaptive stepping. The implementation has been carefully verified comparing numerically determined deflection angles with analytical results presented in the previous section. Note that the distance between source and target plane has been chosen based on the asymptotic behaviour of a hyperbolic-like profile (Eq. (2)).

As a case study, we choose to model the profile of the neutral gas temperature $T=T(r)$ from the core of the microfilament $T(r=0)=T_{1}$ to ambient gas $\left(T_{0}\right)$ with a Gaussian function:

$$
T(r)=T_{0}+\left(T_{1}-T_{0}\right) \exp \left(-\frac{r^{2}}{\sigma^{2}}\right),
$$

where $\sigma$ is the characteristic width of the profile. Note that the optical thickness of the filament is $0.3 \mathrm{~mm}$, while the gas temperature profile exceeds these boundaries significantly. Considering Arago-Biot relation (Eq. 4), then, equation (14) can be transformed to the refractive index and the ray trajectory can be solved numerically using the procedure described above. Moreover, the solution has been evaluated towards the measured maximal deflection angle $\delta_{1}$. This $\delta_{1}$ is related to the core temperature of the filament and with the Gaussian profile more substantiated than in the model based on the hyperbolic-like profile. In Figure 3 the comparison of $\delta_{1}$ for the two model profiles is shown. The dependence between $T_{1}$ and $\delta_{1}$ can be fitted with the equation similar to equations (5) and (8):

$$
\frac{T_{1}}{T_{0}}=\left(1-\frac{\delta_{1}}{C}\right)^{-1}
$$

where $T_{0}$ is the ambient temperature and $C$ is a fitting parameter. Here, the fitting procedure of simulated data based on the Gaussian profile exhibits a satisfying statistic confidence characterized by the predicative power $R^{2}=$ 0.98 of the model given by equation (15) and it leads to an empirical value for the constant $C$, which equals $(0.481 \pm$ $0.002) \operatorname{mrad}$.

\section{Experimental results and discussion}

In the experiment, the effluent of plasma jet operating in the LM3 has been analysed exemplary. In the regime LM3, three filaments rotate along the inner wall of the discharge capillary. The modulated temperature field generated by the discharge filaments in the remote effluent below the outlet of the capillary is evaluated by directing the laser perpendicularly through this region. The laser beam crosses the centre of the jet in radial direction, therefore the deflection of the rays is affected sensitively by the rotating filaments and can be evaluated to provide the maximum temperature below the corresponding filament. The situation is illustrated in Figure 4 where the phases of the deflection signal are linked to the corresponding position of the rotating filament pattern in the jet. Due to 


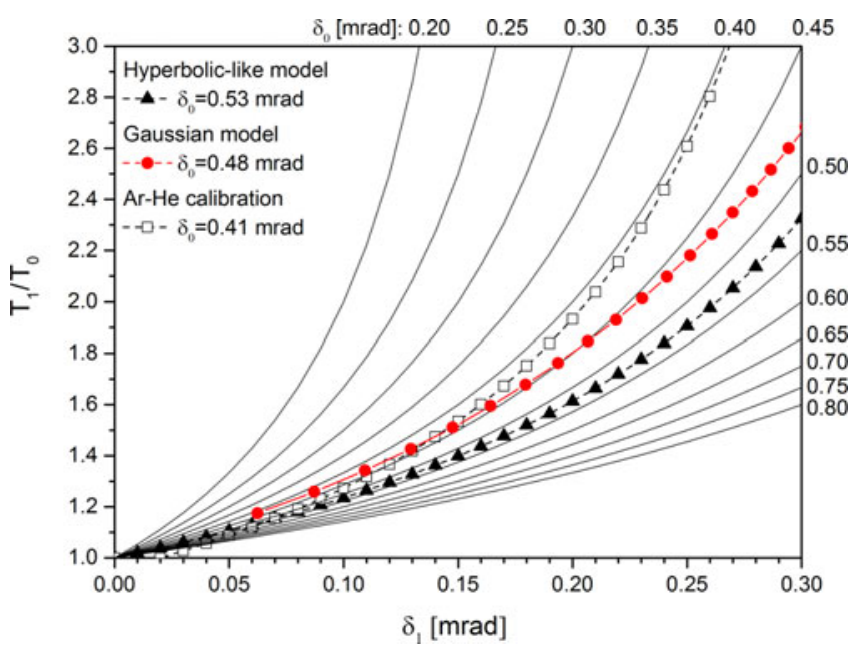

Fig. 3. Characteristics for the determination of the neutral gas temperature of a single filament by LSD.

the symmetric filament pattern the measured frequency of the deflection is three times higher than the rotation frequency of the LM3 which is $\omega / 2 \pi=33 \mathrm{~Hz}$ in this case. Moreover, the deflection signal represents a composition of three components which are partially overlapped. Consequently, the filament rotation projected in the plane perpendicular to the laser beam causes a periodical oscillation of the impact distance of the ray $\rho$ which can be expressed then as: $\rho=\left(D_{\text {in }} / 2-\sigma\right) \sin (3 \omega t+\phi)$, where $\sigma$, $\omega$ and $\phi$ are the characteristic width of the temperature profile, angular velocity of LM3 and phase shift, respectively. They represent fitting parameters in the evaluation of the deflection.

We model the solution of equation (1) for the deflection signal, which can be either measured directly or simulated by the numerical ray tracing, as a product of the maximum deflection $\delta_{1}$ and a shape function of deflection $g$ as:

$$
\delta(\rho)=\delta_{1} \times g=\delta_{0}\left(1-\frac{T_{0}}{T_{1}}\right) \times g(\rho) .
$$

Note that the expression for $\delta_{1}$ is fully consistent with equations (5), (8), and (15) considering the generalization of the respective constants $2\left(n_{0}-1\right), B$ and $C$ which are represented by the parameter $\delta_{0}$. Additionally $g=g(\rho)$ in equation (16) should satisfy the request of normality $g\left(\rho_{1}\right)=1$, where $\rho_{1}$ is an impact distance of the laser ray at the maximum deflection $\delta_{1}$. For the purpose of the experimental data analysis it is convenient to approximate $g(\rho)$ by a suitable analytical expression. It was found that such approximation can be written as:

$$
g(\rho)=\frac{\rho}{\rho_{1}} \exp \left(\frac{\rho_{1}^{2}-\rho^{2}}{2 \rho_{1}^{2}}\right) .
$$

Note that the parameter $\rho_{1}$ is proportional to the width of the neutral gas temperature $\sigma$ introduced in equation (14) and the proportionality coefficient is only slightly dependent on the temperature ratio $T_{1} / T_{0}$. Simulation of the ray propagation shows that for $T_{1}=550 \mathrm{~K}$ the ratio $\rho_{1} / \sigma$ is 1.11. Simulated signals for characteristic temperature

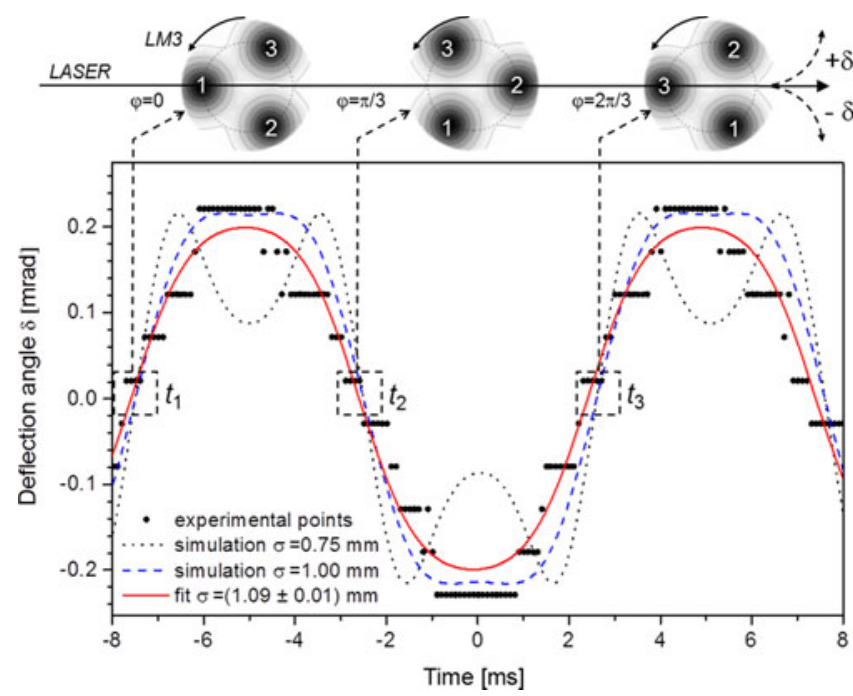

Fig. 4. LSD measurement in the effluent of the plasma jet (LM3) and the simulated deflection calculated for a core filament temperature of $(550 \pm 22) \mathrm{K}$, LM3 frequency $\omega / 2 \pi=$ $100 / 3=33 \mathrm{~Hz}$, and two exemplary widths $\sigma$ of the temperature profile are compared to the fit.

widths $\sigma \in\{0.75,1.00\} \mathrm{mm}$ are compared with experimental data and with the fit obtained from equations (16) and (17). For the fitting procedure (see Fig. 4), we substituted the impact distance $\rho$ in equation (16) by the expression for the LM3 rotation $\rho=\left(D_{\text {in }} / 2-\sigma\right) \sin$ $(3 \omega t+\phi)$ as described before. Here from, the regression parameter $\sigma=(1.09 \pm 0.01) \mathrm{mm}$ is obtained, by setting the temperature $T_{1}$ to $550 \mathrm{~K}$. This value is calculated from the measurement of the maximum deflection $\delta_{1}=0.22 \mathrm{mrad}$ for the LM3 using equation (15) for $\delta_{0}=C=0.48 \mathrm{mrad}$ (see Fig. 3, too). The statistic accuracy of the temperature $T_{1}$ has been calculated to $22 \mathrm{~K}$ from:

$$
\frac{s_{T_{1}}}{T_{1}}=\frac{s_{\delta_{1}}}{\delta_{0}-\delta_{1}}
$$

where $s_{T_{1}}$ and $s_{\delta_{1}}$ are the mean standard deviation of $T_{1}$ and $\delta_{1}$, respectively.

The information on the maximum and width of the temperature profile formed in the effluent directly below the three filaments in LM3 mode $\left(T_{1}=(550 \pm 22) \mathrm{K}\right.$, $\sigma=(1.09 \pm 0.01) \mathrm{mm})$ enables to reconstruct the crosssection of the temperature field at the given axial position in the effluent. The result is shown in Figure 5. Note that the current experiment has been operated stationary, moving neither plasma source nor the laser device. Just the rotating filaments provide the time-dependant deflection. This configuration is highly sensitive to the dynamics of the rotating filaments, however, no information can be extracted on a stationary component of the temperature background directly on the central axis along the diameter of the jet. This constraint can be eliminated by periodic scanning of the whole cross-section of the effluent. The additional benefit of the scanning LSD is that it would allow to measure the stationary modes of the plasma jet, too. This is subject to further cogitation. 


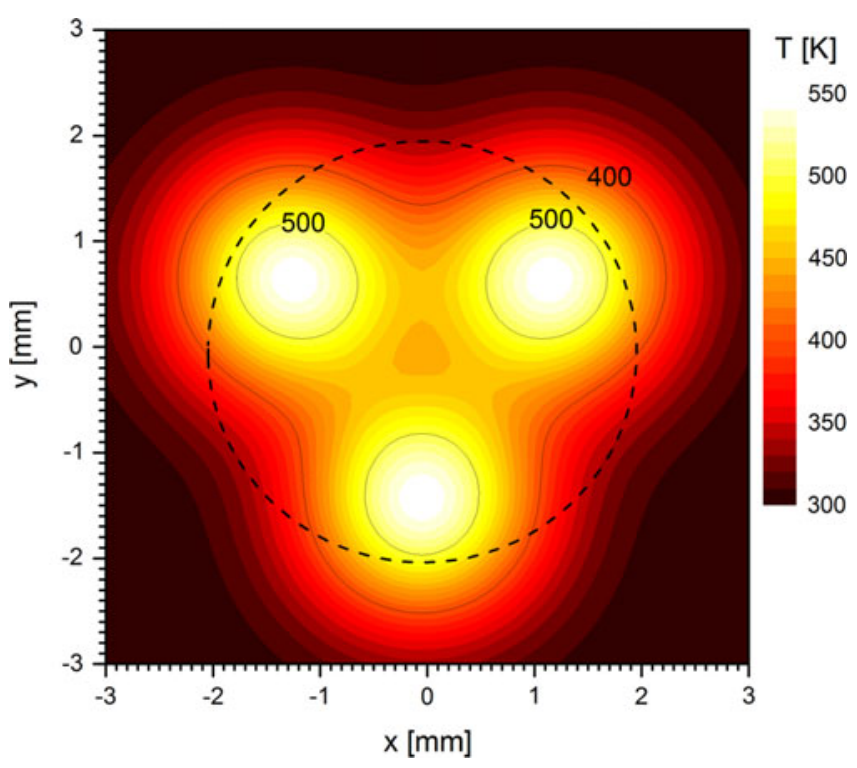

Fig. 5. Dynamic temperature field of the effluent perpendicular to the axis of the plasma jet in LM3 reconstructed by LSD, position: nozzle of the capillary. Dashed line marks the inner wall of the capillary. The period of the pattern rotation is $30 \mathrm{~ms}$.

\section{Conclusion}

The method laser schlieren deflectometry (LSD) discussed here excels with high time and space resolution as compared to conventional methods for determination of the neutral gas temperature in filamentary microdischarges. We derived the principle equation of LSD in three independent ways (Eqs. 5, 8, and 15). The formula can be written in an elegant form as:

$$
\frac{T_{1}}{T_{0}}=\left(1-\frac{\delta_{1}}{\delta_{0}}\right)^{-1},
$$

where $T_{1}$ is the maximum of the neutral gas temperature in the plasma filament, $T_{0}$ is temperature of the optically neutral ambient atmosphere, $\delta_{1}$ is the maximum deflection and $\delta_{0}$ is the characteristic constant of the method. From the to date technical point of view, LSD allows to characterize the neutral gas temperature in the filament core with a time resolution $>10 \mathrm{kHz}$ and spatial resolution of approx. $0.1 \mathrm{~mm}$ which is optimal for parametrizing of a microfilament.

This study validated results of LSD presented in [15] numerically applying the " $F=m a$ " formalism for a light ray, complementary with the experimental calibration and theoretical approach based on the analytic method. Additionally, the outcome of the previous investigations has been extended by implementing a Gaussian profile of the neutral gas temperature. The total deflection of the ray trajectory has been calculated for a series of profile parameters. The obtained results define the dependence (19) at a relative predicative power $R^{2}=0.98$.
Moreover, the numerical approach described in this work opens up the possibility for detailed studies of laser beam deflection by temperature disturbance caused by local, cylindrical plasmas even in remote areas away from active, emitting plasmas. Indeed, it allows to account for real laser beam divergence and intensity profile.

With the actual approach slightly higher neutral gas temperatures (up to $20 \%$ difference) are obtained in the centre of the filament compared to the previously developed hyperbolic-like model [15]. Yet, the obtained temperature difference between the two evaluation approaches remains relatively small as compared to the larger confidence interval of temperature estimates based on other methods (fluorooptic fibre, spectroscopy, etc.), thanks to the high spatial and temporal resolution of LSD. The concept of LSD opens up perspectives for the characterization of more complex multifilamentary patterns and their locally strongly disturbed temperature fields.

Project is supported within the 7th European Framework Programme, project No 316216, "PlasmaShape" and by by DFG, Transregio 24 "Fundamentals of Complex Plasmas", ZB acknowledges support by Project CZ.1.05/2.1.00/03.0086 funded by European Regional Development Fund and project LO1411 (NPU I) funded by Ministry of Education, Youth and Sports of Czech Republic.

\section{References}

1. J. Schäfer, R. Foest, A. Quade, A. Ohl, J. Meichsner, K.-D. Weltmann, Eur. Phys. J. D 54, 211 (2009)

2. J. Schäfer, F. Sigeneger, R. Foest, D. Loffhagen, K.-D. Weltmann, Eur. Phys. J. D 60, 531 (2010)

3. A.P. Papadakis, IEEE Trans. Plasma Sci. 40, 811 (2012)

4. P.R.N. Childs, J.R. Greenwood, C.A. Long, Rev. Sci. Instrum. 71, 2959 (2000)

5. M. Stahl, T. Trottenberg, H. Kersten, Rev. Sci. Instrum. 81, $023504(2010)$

6. J. Voráč, A. Obrusník, V. Procházka, P. Dvořák, M. Talaba, Plasma Sources Sci. Technol. 23, 025011 (2014)

7. M. Blitshteyn, J.M. Zabita, IEEE Trans. Ind. Appl. 24, 745 (1988)

8. A.P. Yalin, Y.Z. Ionikh, R.B. Miles, Appl. Opt. 41, 3753 (2002)

9. V. Hermoch, Czech. J. Phys. B 20, 939 (1970)

10. A. Cristofolini, G. Neretti, F. Roveda, C.A. Borghi, J. Appl. Phys. 111, 033302 (2012)

11. D.A. Feikema, Appl. Opt. 45, 4826 (2006)

12. M.J. Hargather, G.S. Settles, Opt. Lasers Eng. 50, $8(2012)$

13. D. Estruch, N.J. Lawson, D.G. MacManus, K.P. Garry, J.L. Stollery, Rev. Sci. Instrum. 79, 126108 (2008)

14. M. Boselli, V. Colombo, E. Ghedini, M. Gherardi, R. Laurita, A. Liguori, P. Sanibondi, A. Stancampiano, Plasma Chem. Plasma Process. 34, 853 (2014)

15. J. Schäfer, R. Foest, S. Reuter, T. Kewitz, J. Šperka, K.-D. Weltmann, Rev. Sci. Instrum. 83, 103506 (2012) 
16. F.A. Jenkins, H.E. White, Fundamentals of Optics, 4th edn. (McGraw-Hill, New York, 1976)

17. E. Rutherford, J. Philos. Mag. 6, 669 (1911)

18. D.F.T. Arago, J.B. Biot, Mem. Acad. Fr. 7, 301 (1806)

19. H. Landolt, R. Börnstein, W. Meyerhoffer, LandoltBörnstein Physikalisch-Chemische Tabellen (J. Springer, Berlin, 1905)

20. www.POVray.org

21. G. Swartzlander, T.J. Peterson, A.B. Artusio-Glimpse, A.D. Raisanen, Nature Photonics 5, 48 (2011)

22. A.C. Hindmarsh, in Scientific Computing, edited by R.S. Stepleman, vol. 1 of IMACS Transactions on Scientific Computation (North-Holland, Amsterdam, 1983), p. 55
23. J.W. Eaton, D. Bateman, S. Hauberg, R. Wehbring, GNU Octave version 3.8.1 manual: a high-level interactive language for numerical computations (CreateSpace Independent Publishing Platform), ISBN: 1441413006

24. M. Born, E. Wolf, Principles of Optics, 7th edn. (Cambridge University Press, 2003), p. 130

25. J. Evans, M. Rosenquist, Am. J. Phys. 54, 876 (1986)

Open Access This article is distributed under the terms of the Creative Commons Attribution License http:// creativecommons.org/licenses/by/4.0 which permits unrestricted use, distribution, and reproduction in any medium, provided the original author(s) and source are credited. 